\title{
Sur la Condition de E. E. Levi Concernant des Équations Hyperboliques
}

\author{
Par \\ Sigeru Mizohata et Yujiro OHYA*
}

1. Introduction. Il s'agit du problème de Cauchy pour des équations kowalewskiennes à caractéristiques doubles. Nous montrons une condition nécessaire et suffisante pour que ce problème soit bien posé.

Historiquement ce genre de problème a été considéré par E. Levi [6] pour la première fois. Depuis, A. Lax [4] a publié un article sur le même sujet, et M. Yamaguti [11] a étendu ce travail au cas de plusieurs variables. D'autre part, il y a des recherches de Ohya [10] et Leray-Ohya [5] concernant des systèmes kowalewskiens dont les coefficients appartiennent à quelques classes de Gevrey. Très récemment T. Kano [2] a obtenu un résultat concernant la condition nécessaire pour l'équation hyperbolique stricte. Notre tâche est d'étendre le travail de Levi au cas de plusieurs variables, et de donner des résultats plus précis et plus complets que ceux de M. Yamaguti. Nous nous limitons a ésquisser la démonstration. Un article ultérieur donnera la démonstration détaillée et des résultats plus généraux.

2. Soit $P\left(x, t ; \frac{\partial}{\partial x}, \frac{\partial}{\partial t}\right)$ un polynôme différentiel d'ordre $m$ défini dans $\Omega=\left\{(x, t) ; x \in R^{l}, t \in[0, T]\right\}$. Plus précisément

$$
P u=\frac{\partial^{m}}{\partial t^{m}} u+\sum_{\substack{|\nu|+j \leqslant m \\ j<m}} a_{\nu j}\left(x, t ; \frac{\partial}{\partial x}\right) \frac{\partial^{j}}{\partial t^{j}} u
$$

où $a_{\nu j}\left(x, t ; \frac{\partial}{\partial x}\right)$ sont des opérateurs différentiels en $x$ d'ordre $m-j$.

\footnotetext{
Received July 22, 1968.

* Department of Applied Mathematics and Physics, Kyoto University.
} 
Nous supposons que tous les coefficients de $P$ sont indéfiniment différentiables et bornés avec leurs dérivées.

Il s'agit du problème de Cauchy dans $\Omega$;

$$
P\left(x, t ; \frac{\partial}{\partial x}, \frac{\partial}{\partial t}\right) u(x, t)=f(x, t)
$$

avec la donnée de Cauchy portée sur l'hyperplan $t=t_{0}\left(0 \leqslant t_{0} \leqslant T\right)$.

Désignons

$$
D=\frac{1}{i} \frac{\partial}{\partial x}, \quad D_{t}=\frac{1}{i} \frac{\partial}{\partial t}
$$

et écrivons la partie principale de $P$ par

(2.1) $i^{m} P_{m}\left(x, t ; D, D_{t}\right)=i^{m}\left\{D_{t}^{m}+h_{1}(x, t ; D) D_{t}^{m-1}+\cdots+h_{m}(x, t ; D)\right\}$.

Nous supposons que les racines caractéristiques sont au plus doubles et leurs multiplicités sont invariantes par rapport $\dot{a}(x, t, \xi)$. Plus précisément

$$
\begin{aligned}
& \tau^{m}+h_{1}(x, t ; \xi) \tau^{m-1}+\cdots+h_{m}(x, t ; \xi) \\
= & \prod_{i=1}^{s}\left(\tau-\lambda_{i}(x, t ; \xi)\right)^{2} \prod_{j=s+1}^{m-s}\left(\tau-\lambda_{j}(x, t ; \xi)\right),
\end{aligned}
$$

où $\lambda_{1}, \cdots, \lambda_{m-s}$ sont réelles et distinctes, et $\lambda_{1}, \cdots, \lambda_{s}$ sont des racines doubles et $\lambda_{s+1}, \cdots, \lambda_{m-s}$ sont des racines simples.

Comme on sait, on peut associer à la fonction $\lambda_{i}(x, t ; \xi)$, fonction homogène de degré 1 en $\xi$, un opérateur pseudo-différentiel d'ordre 1 (dépendant du paramètre $t$ ) défini par

$$
\lambda_{i}(x, t ; D) f(x)=(2 \pi)^{-l} \int e^{i x \xi} \lambda_{i}(x, t ; \xi) \hat{f}(\xi) d \xi
$$

\section{Posons}

$$
\partial_{i}=D_{t}-\lambda_{i}(x, t ; D)=\frac{1}{i} \frac{\partial}{\partial t}-\lambda_{i}(x, t ; D),
$$

et considérons

$$
\Pi_{m}=\left(\partial_{m-s} \cdots \partial_{s} \cdots \partial_{1}\right)\left(\partial_{s} \partial_{s-1} \cdots \partial_{1}\right)
$$

Remarquons que $P\left(x, t ; \frac{\partial}{\partial x}, \frac{\partial}{\partial t}\right)-\Pi_{m}$ est un opérateur pseudodifférentiel d'ordre $(m-1)$. Désignons 
(2.3) $\quad i^{m-1} C_{m-1}\left(x, t ; D, D_{t}\right)=i^{m}\left\{P_{m}\left(x, t ; D, D_{t}\right)-\Pi_{m}\left(x, t ; D, D_{t}\right)\right\}$ $+i^{m-1} P_{m-1}\left(x, t ; D, D_{t}\right)$,

$C_{m-1}$ est un opérateur différentiel en $t$ dont les coefficients sont des opérateurs pseudo-différentiels en $x$.

Prenons pour base $m$ opérateurs

$$
\begin{aligned}
& 1, \partial_{1}, \partial_{2} \partial_{1}, \cdots, \partial_{s} \partial_{s-1} \cdots \partial_{1}, \partial_{1} \partial_{s} \partial_{s-1} \partial_{s-2} \cdots \partial_{1}, \\
& \cdots, \partial_{m-s-1} \cdots \partial_{s} \cdots \partial_{1} \partial_{s} \cdots \partial_{1} .
\end{aligned}
$$

On peut représenter $C_{m-1}\left(x, t ; D, D_{t}\right)$ sous la forme

$$
\begin{aligned}
& C_{m-1}\left(x, t ; D, D_{t}\right)=c_{m-1}(x, t ; D)+c_{m-2}(x, t ; D) \partial_{1}+c_{m-3} \partial_{2} \partial_{1} \\
& +\cdots+c_{m-s} \partial_{s-1} \partial_{s-2} \cdots \partial_{1}+\cdots+c_{0} \partial_{m-s-1} \cdots \partial_{1} \partial_{s} \cdots \partial_{1} \\
& +Q_{m-2}\left(x, t ; D, D_{t}\right),
\end{aligned}
$$

où $c_{m-i}(x, t ; D)(i=1,2, \cdots, m-1)$ est opérateur pseudo-différentiel dont le symbole $c_{m-i}(x, t ; \xi)$ est homogène de degré $(m-i)$ en $\xi$, et $Q_{m-2}$ est d'ordre $(m-2)$. Comme on verra dans la section $3, c_{m-i}$ sont déterminés de la manière unique.

Introduisons la condition suivante :

(2.6) Condition A. Tous les symboles $c_{m-i}(x, t ; \xi)(i=1,2, \cdots, s)$ s'annulent identiquement pour $(x, t ; \xi) \in \Omega \times R_{\xi}^{l}$.

Démontrons le

Théorème 1. Sous la condition A, le problème de Cauchy est uniformément bien posé.

Démonstration (en bref).

Compte tenu de (2.3), l'équation

$$
P\left(x, t ; \frac{\partial}{\partial x}, \frac{\partial}{\partial t}\right) u(x, t)=f(x, t)
$$

s'écrit

$$
\left[i^{m} \Pi_{m}+i^{m-1} C_{m-1}\right] u+R_{m-2} u=f,
$$

où $R_{m-2}$ est d'ordre $m-2$. On considère d'abord l'équation

$$
\left[\Pi_{m}-i C_{m-1}\right] u(x, t)=i^{-m} f(x, t) .
$$

Posons 


$$
\left(u, \partial_{1} u, \partial_{2} \partial_{1} u, \cdots, \partial_{m-s-1} \cdots \partial_{1} u\right)=\left(u_{0}, u_{1}, \cdots, u_{m-1}\right)
$$

et notons

$$
U={ }^{t}\left(u_{0}, u_{1}, \cdots, u_{m-1}\right) .
$$

L'équation (2.8) peut s'écrire sous la forme matricielle

$$
\begin{aligned}
& D_{t} U(x, t)=H(x, t ; D) U(x, t)+F(x, t) .
\end{aligned}
$$

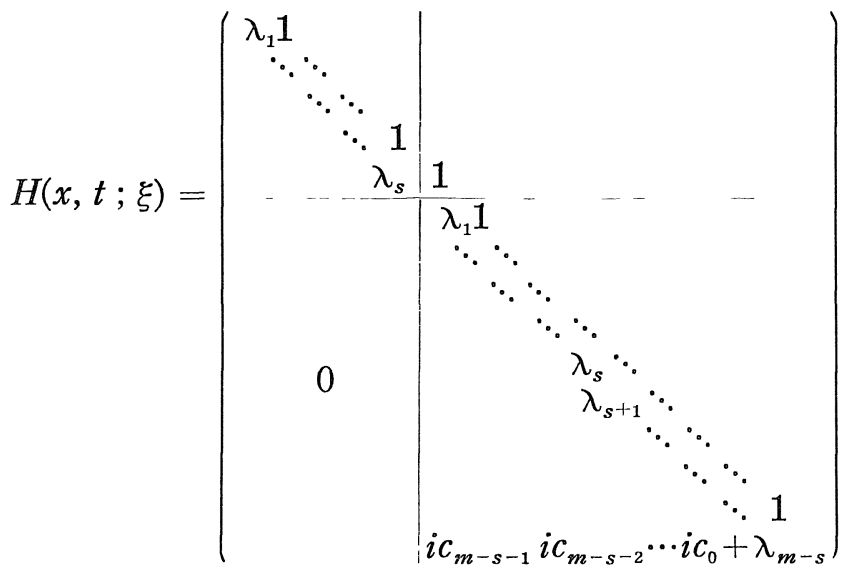

où $\lambda_{i}=\lambda_{i}(x, t ; \xi)$ (homogène de degré 1 en $\left.\xi\right), c_{m-i}=c_{m-i}(x, t ; \xi)$ (homogène de degré $(m-i)$ en $\xi$ ). Enfin $F={ }^{t}\left(0, \cdots, 0, i^{-m} f\right)$.

Finalement posons

$$
V(x, t)={ }^{t}\left((\Lambda+1)^{m-2} u_{0}, \cdots,(\Lambda+1)^{m-s-1} u_{s-1},(\Lambda+1)^{m-s-1} u_{s}, \cdots, u_{m-1}\right) .
$$

Remarquons que nous avons mis le même poids aux deux composantes $u_{s-1}$ et $u_{s}$. Grâce à cet artifice, et compte tenu des degrés de $c_{m-i}$, on peut écrire (2.10) sous la forme

$$
D_{t} V(x, t)=H_{0}(x, t ; D) \Lambda V(x, t)+B(x, t ; D) V(x, t)+F(x, t)
$$

où

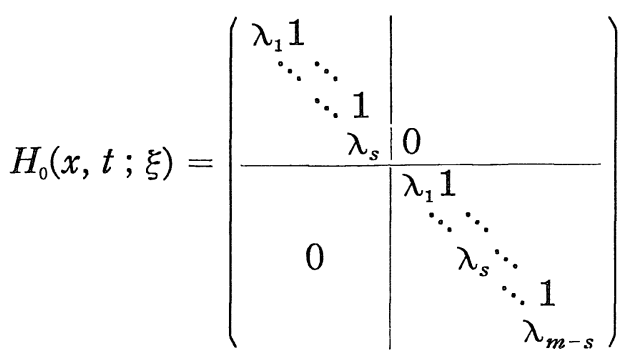


où $\lambda_{i}=\lambda_{i}(x, t ; \xi /|\xi|)$ et $B$ est un opérateur borné dans $\left(L^{2}\left(R^{l}\right)\right)^{m}$.

$H_{0}(x, t ; D) \Lambda$ est donc la somme directe de deux matrices regulièrement hyperboliques. Ce système est déjà considéré par plusieurs auteurs.

Etant donnés la donée initiale $V\left(x, t_{0}\right) \in \mathscr{D}_{L^{2}}^{k^{2}}\left(0 \leqslant t_{0} \leqslant T\right)$ et le second membre $F(x, t) \in \mathcal{E}_{t}{ }^{0}\left(\mathscr{D}_{L^{2}}^{k}\right)$, il existe une solution unique $V(x, t)$ de (2.11), une fois continuement différentiable dans $\mathscr{D}_{L^{2}}^{k-1}$ $(k=1,2, \cdots)$, et on a

$$
\|V(t)\|_{k} \leqslant C(T)\left[\left\|V\left(t_{0}\right)\right\|_{k}+\int_{t_{0}}^{t}\|F(s)\|_{k} d s\right] .
$$

Prenons par exemple $k=1$. Alors $\left(u_{0}(x, t), u_{1}(x, t), \cdots, u_{s-1}(x, t)\right.$, $\left.u_{s}(x, t), \cdots, u_{m-1}(x, t)\right)$ est continu dans $\mathscr{D}_{L^{2}}^{n-1} \times \mathscr{D}_{L^{2}}^{m} \overline{2}^{2} \times \cdots \times \mathscr{D}_{L^{2}}^{m} \times \cdots$ $\cdots \times \mathscr{D}_{L^{2}}^{1}$, et continuement différentiable dans $\mathscr{D}_{L^{2}}^{m} \overline{2}^{2} \times \mathscr{D}_{L^{2}}^{n-3} \times \cdots$ $\cdots \times \mathscr{D}_{L^{2}}^{m-s-1} \times \mathscr{D}_{L^{m}}^{m-s-1} \times \cdots \times L^{2}$. Cela implique à fortiori que $(u(x, t)$, $\left.\frac{\partial}{\partial t} u(x, t), \cdots, \frac{\partial^{m-1}}{\partial t^{m-1}} u(x, t)\right)$ est continu dans $\mathscr{D}_{L^{2}}^{m-1} \times \mathscr{D}_{L^{2}}^{m-2} \times \cdots \times L^{2}$.

Ensuite si l'on désigne

$$
\|\mid u(x, t)\|^{2}=\|u(x, t)\|_{m-1}^{2}+\left\|\frac{\partial}{\partial t} u(x, t)\right\|_{m-2}^{2}+\cdots+\left\|\frac{\partial^{m-1}}{\partial t^{m-1}} u(x, t)\right\|_{0}^{2},
$$

on a

$$
\||| u(x, t)\| \mid \leqslant \widetilde{C}(T)\left(\left|\left\|(\Lambda+1) u\left(x, t_{0}\right) \mid\right\|+\int_{t_{0}}^{t}\|f(x, s)\|_{1} d s\right) .\right.
$$

Cette inégalité nous permet de résoudre le problème de Cauchy pour (2.7) par approximation successive. En effet, on a

$$
\text { i| } R_{m-2} u(x, t)\left\|_{1} \leqslant C^{\prime}\right\||u(x, t)| \| .
$$

Résumons ce qu'on a obtenu: Considérons l'équation (2.7). Pour toute donnée initiale à $t=t_{0}$ et le second membre $f(x, t)$ tels que $\left(u_{0}(x), u_{1}(x), \cdots, u_{m-1}(x)\right) \in \mathscr{D}_{I^{2}}^{m+p} \times \mathscr{D}_{L^{2}}^{m+p-1} \times \cdots \times \mathscr{D}_{L^{2}}^{p+1} f(x, t) \in \mathcal{E}_{t}{ }^{0}\left(\mathscr{D}_{L^{2}}^{p+1}\right)$, il existe une solution unique $u(x, t)$ telle que $\left(u(x, t), \cdots, \frac{\partial^{m-1}}{\partial t^{m-1}} u(x, t)\right)$ soit continue dans $\mathscr{D}_{L^{2}}^{m+p-1} \times \cdots \times \mathscr{D}_{L^{2}}^{p}(p=0,2, \cdots)$.

3. Condition de Levi. Nous allons exprimer la condition (A) sous une forme explicite. Pour cela remarquons que, compte tenu de (2.5), si l'on désigne le symbole principal de $C_{m-1}$ (la partie homogène 
d'ordre $(m-1))$ par $\sigma_{m-1}\left(C_{m-1}\right)$, on a

$$
c_{m-1}(x, t ; \xi)=\left.\sigma_{m-1}\left(C_{m-1}\left(x, t ; D, D_{t}\right)\right)\right|_{\tau=\lambda_{1}(x, t ; \xi)} .
$$

Ceci remarqué, soit

$$
\Pi_{m}^{(1)}=\left(\partial_{m-s} \cdots \partial_{s} \cdots \partial_{2}\right)\left(\partial_{s} \cdots \partial_{2}\right) \partial_{1}^{2} .
$$

Alors,

$$
\begin{aligned}
& \Pi_{m}\left(x, t ; D, D_{t}\right)-\Pi_{m}^{(1)}\left(x, t ; D, D_{t}\right) \\
= & \left(\partial_{m-s} \cdots \partial_{2}\right)\left(\partial_{1} \partial_{s} \cdots \partial_{2}-\partial_{s} \cdots \partial_{2} \partial_{1}\right) \partial_{1}
\end{aligned}
$$

montre que la partie homogène d'ordre $(m-1)$ du symbole $\sigma_{m-1}\left(\Pi_{m}-\Pi_{m}^{(1)}\right)$ est divisible par $\left(\tau-\lambda_{1}(x, t ; \xi)\right)$. On a donc

$$
\begin{aligned}
\sigma_{m-1}\left(C_{m-1}\left(x, t ; D, D_{t}\right)\right) \equiv & i \sigma_{m-1}\left(P_{m}\left(x, t ; D, D_{t}\right)-\Pi_{m}^{(i)}\left(x, t ; D, D_{t}\right)\right) \\
& +P_{m-1}(x, t ; \xi, \tau) \quad\left(\bmod \left(\tau-\lambda_{1}\right)\right) .
\end{aligned}
$$

Ensuite, si l'on désigne

$$
\Pi_{m-1}^{(1)}=\left(\partial_{m-s} \circ \cdots \circ \partial_{s} \circ \cdots \circ \partial_{2}\right) \circ\left(\partial_{s} \circ \cdots \circ \partial_{2} \circ \partial_{1}\right),
$$

on aura, par la même raison

$$
\begin{aligned}
& \sigma_{m-1}\left(P_{m}\left(x, t ; D, D_{t}\right)-\Pi_{m-1}^{(1)}\left(x, t ; D, D_{t}\right) \partial_{1}\right) \\
\equiv & \sigma_{m-1}\left(\Pi_{m-1}^{(1)} \circ \partial_{1}-\Pi_{m-1}^{(1)} \partial_{1}\right) \quad\left(\bmod \left(\tau-\lambda_{1}\right)\right) .
\end{aligned}
$$

Or, on sait que

$$
\begin{aligned}
& \sigma_{m-1}\left(\Pi_{n-1}^{(1)} \circ \partial_{1}-\Pi_{m-1}^{(1)} \partial_{1}\right) \\
= & i^{-1}\left(\frac{\partial}{\partial \tau} \Pi_{m-1}^{(1)} \frac{\partial \lambda_{1}}{\partial t}+\sum_{\alpha=1}^{l} \frac{\partial}{\partial \xi_{\alpha}} \Pi_{m-1}^{(1)} \frac{\partial \lambda_{1}}{\partial x_{\alpha}}\right) .
\end{aligned}
$$

Cette formule s'est montrée pour la première fois par Matsumura (voir [7], Proposition 2. 1, voir aussi [3]).

D'autre part,

$$
P_{m}(x, t ; \xi, \tau)=\left(\tau-\lambda_{1}(x, t ; \xi)\right)^{2} \Pi\left(\tau-\lambda_{j}(x, t ; \xi)\right)
$$

montre que, si l'on désigne $P_{m}^{\prime}(x, t ; \xi, \tau)=\frac{\partial}{\partial \tau} P_{m}$,

$$
\begin{aligned}
& \left.\frac{\partial}{\partial \tau} \Pi_{m-1}^{(1)}(x, t ; \xi, \tau)\right|_{\tau=\lambda_{1}}=\left.\frac{1}{2} \frac{\partial}{\partial \tau} P_{m}^{\prime}(x, t ; \xi, \tau)\right|_{\tau=\lambda_{1}} \\
& \left.\frac{\partial}{\partial \xi_{\alpha}} \Pi_{m-1}^{(1)}(x, t ; \xi, \tau)\right|_{\substack{\tau=\lambda_{1} \\
\tau}}=\left.\frac{1}{2} \frac{\partial}{\partial \xi_{\alpha}} P_{m}^{\prime}(x, t ; \xi, \tau)\right|_{\tau=\lambda_{1}} .
\end{aligned}
$$


D'où

(3. 6)

$$
\begin{aligned}
& \left.\sigma_{m-1}\left(C_{m-1}\left(x, t ; D, D_{t}\right)\right)\right|_{\tau=\lambda_{1}} \\
= & P_{m-1}\left(x, t ; \xi, \lambda_{1}(x, t ; \xi)\right)+\left.\frac{1}{2}\left[\frac{\partial}{\partial \tau} P_{m}^{\prime} \frac{\partial}{\partial t} \lambda_{1}+\sum_{\alpha=1}^{l} \frac{\partial}{\partial \xi_{\alpha}} P_{m}^{\prime} \frac{\partial}{\partial x_{\infty}} \lambda_{1}\right]\right|_{\tau=\lambda_{1}} .
\end{aligned}
$$

On est ramené à définir les fonctions suivantes.

Définition 1. Soient $\lambda_{j}$ des racines doubles. Nous appelons la fonction de Levi attachée $\dot{a} \lambda_{j}$ celle définie par

$$
\begin{aligned}
& L_{j}(x, t ; \xi)=\left[P_{m-1}(x, t ; \xi, \tau)+\frac{1}{2}\left(\frac{\partial}{\partial \tau} P_{m}^{\prime} \frac{\partial}{\partial t} \lambda_{j}+\sum_{\alpha=1}^{l} \frac{\partial}{\partial \xi_{\alpha}} P_{m}^{\prime} \frac{\partial}{\partial x_{\alpha}} \lambda_{j}\right)\right]_{\tau=\lambda_{i}} \\
& \text { où } \quad P_{m}^{\prime}=\frac{\partial}{\partial \tau} P_{m}(x, t ; \xi, \tau) .
\end{aligned}
$$

Résumons ce qu'on a obtenu: Pour que $c_{m-1}(x, t ; \xi) \equiv 0$, il faut et il suffit qu'on ait $L_{1}(x, t ; \xi) \equiv 0$.

On a le

Lemme 3.1. Soit $C_{m-1}^{0}(x, t ; \xi, \tau)$ le symbole principal de $C_{m-1}\left(x, t ; D, D_{t}\right)$, on $a$, pour $j=1,2, \cdots, s$,

$$
\begin{aligned}
L_{j}(x, t ; \xi)= & C_{m-1}^{0}\left(x, t ; \xi, \lambda_{j}(x, t ; \xi)\right) \\
= & c_{m-1}+c_{m-2}\left(\lambda_{j}-\lambda_{1}\right)+c_{m-3}\left(\lambda_{j}-\lambda_{1}\right)\left(\lambda_{j}-\lambda_{2}\right)+\cdots \\
& \quad+c_{m-j}\left(\lambda_{j}-\lambda_{1}\right)\left(\lambda_{j}-\lambda_{2}\right) \cdots\left(\lambda_{j}-\lambda_{j-1}\right) .
\end{aligned}
$$

D'où

Lemme 3.2. Soit $\left(x_{0}, t_{0} ; \xi_{0}\right) \in \Omega \times R^{l}-\{0\}$. Pour que

$$
L_{j}\left(x_{0}, t_{0} ; \xi_{0}\right)=0 \quad(j=1,2, \cdots, s),
$$

il faut et il suffit qu'on ait

$$
c_{m-i}\left(x_{0}, t_{0} ; \xi_{0}\right)=0 \quad(i=1,2, \cdots, s) .
$$

On a donc le

Théorème 2. Pour que l'opérateur $\left(P_{m}, P_{m-1}\right)$ satisfasse ḋ la condition $A$, il faut et il suffit qu'on ait

$$
L_{j}(x, t ; \xi) \equiv 0 \quad(j=1,2, \cdots, s) .
$$


4. Domaine d'influence. Faisons le changement de coordonnées "space-like", de $(x, t)$ à $\left(x^{\prime}, t^{\prime}\right)$ tel que

$$
t^{\prime}=\varphi(x, t), \quad x_{\alpha}^{\prime}=x_{\alpha} \quad(1 \leqslant \alpha \leqslant l)
$$

alors

$$
\frac{\partial}{\partial t}=\varphi_{t} \frac{\partial}{\partial t^{\prime}}, \quad \frac{\partial}{\partial x_{\alpha}}=\varphi_{x_{\alpha}} \frac{\partial}{\partial t^{\prime}}+\frac{\partial}{\partial x_{\alpha}{ }^{\prime}},
$$

qui implique $(\xi, \tau)$ se transforme à $\left(\varphi_{x} \tau+\xi, \varphi_{t} \tau\right)$ d'après [3] et [4], où l'on a suppréssé pour faciliter l'écriture.

Note. La transformation (4.1) est dit "space-like" quand $\lambda_{i}(x, t ; \xi)$ étant des racines caractéristiques de $P$,

$$
\left(\frac{\partial \varphi}{\partial t}\right)^{2}-\lambda_{\max }^{2} \sum_{\alpha=1}^{7}\left(\frac{\partial \varphi}{\partial x_{\alpha}}\right)^{2}>0
$$

où

$$
\lambda_{\max }=\sup _{\substack{(x, t) \in \Omega \\|\xi|=1}}\left|\lambda_{i}(x, t ; \xi)\right| \quad \text { pour } 1 \leqslant i \leqslant m-s .
$$

\section{Lemme 4. 1.}

(4.3) $\partial_{i}=D_{t}-\lambda_{i}(x, t ; D)$ se transforme $\dot{a}$

$$
\begin{aligned}
& \left(\varphi_{t}-\lambda_{i}\left(x, t ; \varphi_{x}\right)\right) \psi_{i}\left(x, t ; D, D_{t}\right)\left(D_{t}-\mu_{i}(x, t ; D)\right) \\
& +e_{i}\left(x, t ; D, D_{t}\right),
\end{aligned}
$$

où $\psi_{i}(x, t ; \xi, \tau)$ est homogène de degré 0 en $(\xi, \tau)$ tel que $\prod_{i=1}^{m} \psi_{i}(x, t ; \xi, \tau) \equiv 1$ et indéfiniment différentiables en dehors de l'origine; $e_{i}\left(x, t ; D, D_{t}\right)$ est un opérateur pseudo-différentiel d'ordre 0.

Preuve. Considérons l'équation en $\tau$

$$
\varphi_{t} \tau-\lambda_{i}\left(x, t ; \varphi_{x} \tau+\xi\right)=0,
$$

subordonnée au changement de coordonnées, compte tenu de ce que le symbole de $\partial_{i}$ est $\tau-\lambda_{i}(x, t ; \xi)$. Si l'on note cette racine par $\mu_{i}(x, t ; \xi)$, homogène de degré 1 en $\xi$ pour $(x, t)$ et $\xi$ fixés, alors l'allure du premier membre de (4.4) pour $\tau \rightarrow \infty$ montre q'elle équivaut à

$$
\left(\varphi_{t}-\lambda_{i}\left(x, t ; \varphi_{x}\right)\right) \psi_{i}(x, t ; \xi, \tau)\left(\tau-\mu_{i}(x, t ; \xi)\right)=0
$$


D'autre part, si l'on désigne $\widetilde{P}_{m}$ la transformée de $P_{m}$ par (4.1), alors on aura

$$
\widetilde{P}_{m}=P_{m}\left(x, t ; \varphi_{x} \tau+\xi, \varphi_{t} \tau\right)=P_{m}\left(x, t ; \varphi_{x}, \varphi_{t}\right) \prod_{i=1}^{m}\left(\tau-\mu_{i}\right)
$$

qui montre $\prod_{i=1}^{m} \psi_{i}(x, t ; \xi, \tau) \equiv 1$. Enfin, en employant la formule de moyenne, il suffit de déduire de (4.4) la relation

$$
\left(\varphi_{t}-\sum_{\alpha=1}^{l} \frac{\partial \lambda_{i}}{\partial \xi_{\alpha}}\left(x, t ; \varphi_{x} \nu_{i}+\xi\right) \frac{\partial \varphi}{\partial x_{\alpha}}\right)\left(\tau-\mu_{i}(x, t ; \xi)\right)=0
$$

$\nu_{i}$ éxistant au voisinage de $\tau=\mu_{i}(x, t ; \xi)$.

Ceci prouve que la condition $A$ se conserve par le changement de coordonnées (4.1); en effet, sous cette condition, notre problème de Cauchy se ramène à $(2.7)$, où $\Pi_{m}$ et $C_{m-1}$ sont donnés par (2.3) et (2.5) tel que $c_{m-i}(x, t ; \xi) \equiv 0(1 \leqslant i \leqslant s)$. Alors, le lemme 4.1 nous indique que, après la transformation (4.1), les symboles de degré $m$ et $m-1$ de $\widetilde{P}$ sont respectivement

$$
\prod_{i=1}^{s}\left\{\left(\varphi_{t}-\lambda_{i}\left(x, t ; \varphi_{x}\right)\right)\left(\tau-\mu_{i}\right)\right\}_{j=s+1}^{2 m-s}\left(\varphi_{t}-\lambda_{j}\left(x, t ; \varphi_{x}\right)\right)\left(\tau-\mu_{i}\right)
$$

et

$$
\begin{aligned}
\sum_{i=1}^{m} e_{i}(x, t ; \xi, \tau) \frac{\widetilde{P}_{m}}{\psi_{i}\left(\varphi_{t}-\lambda_{i}\left(x, t ; \varphi_{x}\right)\right)\left(\tau-\mu_{i}\right)}+\sum_{i=0}^{m-s-1} \widetilde{c}_{m-s-i-1}(x, t ; \xi) \\
\quad \times \prod_{j=1}^{i} \psi_{j}\left(\varphi_{t}-\lambda_{j}\left(x, t ; \varphi_{x}\right)\right)\left(\tau-\mu_{j}\right) \prod_{k=1}^{s} \psi_{k}\left(\varphi_{t}-\lambda_{k}\left(x, t ; \varphi_{x}\right)\right)\left(\tau-\mu_{k}\right)
\end{aligned}
$$

où $\tilde{c}_{m-s-1-i}$ sont les transformés des $c_{m-s-1-i}(x, t ; D)$. Ceci montre qu'ils contiennent respectivement des facteurs $\prod_{i=1}^{s}\left(\tau-\mu_{j}\right)^{2}$ et $\prod_{i=1}^{s}\left(\tau-\mu_{j}\right)$; ce qui prouve notre énoncé. Or, nous obtenons le

Théorème 3. La solution du problème de Cauchy

$$
P u=0 \quad \text { avec }\left(\frac{\partial}{\partial t}\right)^{i} u\left(x, t_{0}\right)=\varphi_{i}(x) \quad 0 \leqslant i \leqslant m-1
$$

a son support dans

$$
\begin{aligned}
& \left\{x ; \underset{\xi}{\cup}|x-\xi| \leqslant \lambda_{\max }\left|t-t_{0}\right|, \xi\right. \text { parcourant le support } \\
& \text { de } \left.\left(\varphi_{0}(x), \cdots, \varphi_{m-1}(x)\right)\right\} \text { pour chaque } t .
\end{aligned}
$$

Puisque le Théorème 1 s'applique au problème de Cauchy transformé 
par (4.1) ceci suffit à prouver le théorème d'unicite locale et donc le Théorème ci-dessus concernant le domaine d'influence.

5. Nous allons montrer la réciproque du Théorème 1 . Pour simplifier notre raisonnement, nous supposons que

$$
P_{m-1}\left(x, t ; \frac{\partial}{\partial x}, \frac{\partial}{\partial t}\right) \text { soit } \dot{a} \text { coefficients réels. }
$$

Enonçons

Théorème 4. Pour que le problème de Cauchy soit bien posé, dans $\Omega=R^{l} \times[0, T]$, il faut qu'on ait

$$
L_{j}(x, t ; \xi) \equiv 0 \quad(j=1,2, \cdots, s) .
$$

Puisque la démonstration est délicate, nous nous limitons à indiquer le point essentiel. Pour montrer le Théorème 4, nous utiliserons la méthode employée dans [9]. Nous supposons que le problème de Cauchy soit bien posé et qu'une au moins des $L_{j}$ ne soit pas identiquement nulle, et montrerons que ces deux hypothèses nous conduisent à une contradiction.

Soit

$$
P u=f .
$$

Prenons l'origine et supposons qu'une au moins des $L_{j}(0,0 ; \xi)$ ne soit pas identiquement 0 . Alors en prenant $\left(x_{0}, t_{0}\right)$ et son voisinage convenablement, on peut supposer que les $L_{j}$ soient classées en deux groupes ;

1) il existe un $\xi_{0}\left(\left|\xi_{0}\right|=1\right)$, tel que $L_{i}\left(x_{0}, t_{0} ; \xi_{0}\right) \neq 0$,

2) $L_{i}(x, t ; \xi)=0$ pour $(x, t) \in V$, et $\xi \in R^{l}$.

Par un artifice, on voit qu'il suffit de considérer le cas où 2) ne se présente pas.

Ceci supposé, on considère le problème de Cauchy avec le temps initial $t_{0}$. On peut supposer encore que $\left(x_{0}, t_{0}\right)$ soit à l'origine. Alors notre hypothèse devient: $L_{j}(x, t ; \xi)$ sont des fonctions à valeurs réelles, et

$$
\left|L_{j}(x, t ; \xi)\right| \geqslant \delta \quad(j=1,2, \cdots, s)(\delta>0)
$$


pour tout $(x, t) \in V$, et tout $\xi \in W\left((0,0) \in V, \xi_{0} \in W\right)$.

Nous allons effectuer deux procédés de localisations. D'abord, soit $\beta(x) \in \mathscr{D}(\beta(0) \neq 0)$ dont le support est petit. Partons de

$$
P\left(x, t ; \frac{\partial}{\partial x}, \frac{\partial}{\partial t}\right) u(x, t)=0 .
$$

Alors

$$
P(\beta u)-\sum_{\mid \sigma i \geqslant 1} P^{(\sigma)}\left(\beta^{(\sigma)} u\right)=0, \quad \text { (d'après Leibniz) . }
$$

Sous cette forme, on peut changer les coefficients de $P\left(x, t ; \frac{\partial}{\partial x}, \frac{\partial}{\partial t}\right)$ en dehors du support de $\beta$. Ensuite soit $\alpha(\xi) \in \mathscr{D}\left(R^{l}\right)(0 \leqslant \alpha(\xi) \leqslant 1)$ dont le support soit contenu dans $W$, et égale à 1 au voisinage de $\xi_{0}$. En désignant $\alpha_{n}(\xi)=\alpha(\xi / n)$, on définit l'opérateur $\alpha_{n}(D) f$ pour toute $f \in L^{2}$, par la transformation de Fourier :

$$
\left(\alpha_{n}(D) f\right)^{\wedge}=\alpha_{n}(\xi) \hat{f}(\xi) .
$$

En appliquant $\alpha_{n}(D)$ à (5.3) à gauche, on aura

$$
P\left[\alpha_{n}(D) \beta u\right]=\sum_{\substack{1 \leqslant|\rho+\sigma| \leqslant k k \\|\rho| \leqslant k}} Q_{\rho_{\sigma}}\left(x, t ; D, D_{t}\right)\left[\alpha_{n}^{(\rho)}(D) \beta^{(\sigma)} u\right]+R_{k}[u]
$$

où

1) $Q_{\rho_{\sigma}}$ est un opérateur différentiel d'ordre $(m-|\sigma|)$, et en particulier, $Q_{e i 0}=i^{m} \frac{\partial}{\partial x_{\alpha}} P\left(x, t ; D, D_{t}\right)$ et $Q_{0 e i}=-i^{m} \frac{\partial}{\partial \xi_{i}} P\left(x, t ; D, D_{t}\right)$

2) $R_{k}$, plus précisément $R_{k}^{(n)}$ satisfait à l'inégalité de la forme

$$
\left\|R_{k}^{(n)}[u(x, t)]\right\| \leqslant \frac{c}{n^{k-l}}\|u(x, t)\| \|
$$

où $\|\cdot\|$ signifie la $L^{2}$-norme prise dans l'espace des $x$, et

$$
|:| u\left|\left\|^{2}=\right\| D_{t}^{m-1} u\left\|^{2}+|| D_{t}^{m-2} u\right\|_{1}^{2}+\cdots+\| u\right|_{m-1}^{2} .
$$

Dans (5.5), si l'on utilise l'expression

$$
P_{m}+P_{m-1}=\Pi_{m}-i C_{m-1},
$$

on peut utiliser $\partial_{i}=D_{t}-\lambda_{i}(x, t ; D)$ sous des formes modiffiées, c'està-dire que l'oscillation de $\lambda_{i}(x, t ; \xi)$ peut ètre supposée petite. Plus 
précisément, grâce à deux localisations, on suppose que $\mid \lambda_{i}(x, t ; \xi)-$ $\lambda_{i}\left(0,0 ; \xi_{0}\right) \mid$ soit petite pour tout $(x, t) \in \Omega$ et $\xi \in S$ (sphère unité). Il en est de même de $C_{m-1}(x, t ; \xi, \tau)$.

Ceci remarqué, considérons

$$
P\left[\alpha_{n}(D) u\right]=f
$$

C'est-à-dire, d'après (2.7),

$$
\left(\Pi_{m}-i C_{m-1}\right)\left[\alpha_{n}(D) u\right]+i^{-m} R_{m-2}\left[\alpha_{n}(D) u\right]=i^{-m} f .
$$

On est ramené à considérer le système suivant:

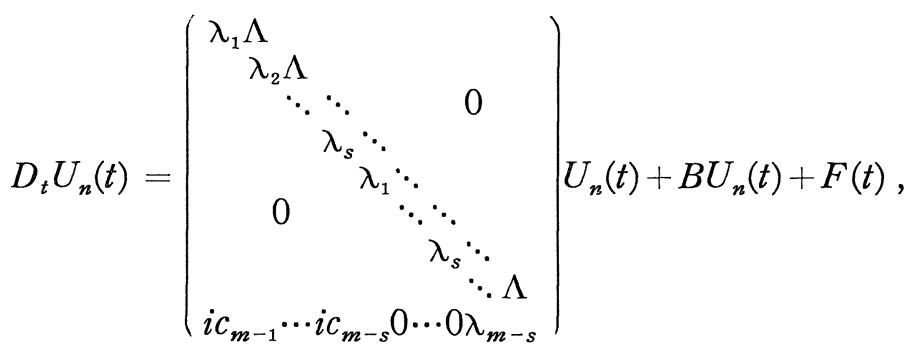

ou plus simplement

$$
D_{t} U_{n}=H(x, t ; D) U_{n}+B U_{n}+F,
$$

où

1) $U_{n}(t)={ }^{t}\left((\Lambda+1)^{m-1}\left[\alpha_{n} u\right],(\Lambda+1)^{m-2} \partial_{1}\left[\alpha_{n} u\right],(\Lambda+1)^{m-3} \partial_{2} \partial_{1}\left[\alpha_{n} u\right]\right.$,

$$
\left.\cdots, \partial_{m-s-1} \cdots \partial_{1} \partial_{s} \cdots \partial_{1}\left[\alpha_{n} u\right]\right) \text {. }
$$

2) $\lambda_{i}=\lambda_{i}(x, t ; D), \sigma\left(c_{m-j}(x, t ; D)\right)=c_{m-j}(x, t ; \xi /|\xi|)$

3)

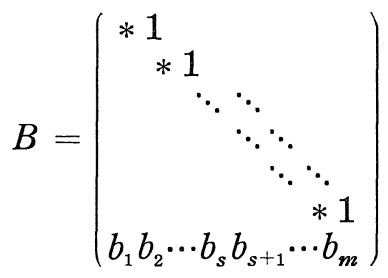

où $*$ sont des opérateurs pseudo-différentiels d'ordre 0 , et $b_{1}, \cdots, b_{s}$ sont des opérateurs pseudo-différentiels d'ordre $(-1)$ et $b_{s+1}, \cdots, b_{m}$ sont d'ordre 0 .

4) $F={ }^{t}\left(0, \cdots, 0, i^{-m} f\right)$. 


\section{Considérons}

$$
\begin{aligned}
\operatorname{det} & (\lambda I-H(x, t ; \xi))=\prod_{i=1}^{s}\left(\lambda-\lambda_{i}(x, t ; \xi)\right)_{i=s+1}^{2}\left(\lambda-\lambda_{j}(x, t ; \xi)\right) \\
& -i\left[c_{m-1}+c_{m-2}\left(\lambda-\lambda_{1}\right)+\cdots+c_{m-s}\left(\lambda-\lambda_{1}\right) \cdots\left(\lambda-\lambda_{s-1}\right)\right]=0 .
\end{aligned}
$$

Alors, les racines perturbées peuvent être développées, soit par Puiseux ou bien par Taylor. En particulier

$$
\lambda_{j}^{ \pm}(x, t ; \xi)=\lambda_{j}(x, t ; \xi) \pm\left[i L_{j}(x, t ; \xi) / \prod_{k \neq j}\left(\lambda_{j}-\lambda_{k}\right)^{2} \prod_{i=s+1}^{m-s}\left(\lambda_{j}-\lambda_{i}\right)\right]^{1 / 2}+\cdots
$$

où

$$
\begin{aligned}
& -\operatorname{Im} \lambda_{j}^{+}(x, t ; \xi)>\delta^{\prime}|\xi|^{1 / 2} \\
& -\operatorname{Im} \lambda_{j}^{-}(x, t ; \xi)<-\delta^{\prime}|\xi|^{1 / 2}
\end{aligned}
$$

pour $|\xi|$ assez grand.

Compte tenu de ce fait, on définit le normalisateur $N(x, t ; \xi)$ de $H(x, t ; \xi)$ de la manière suivante; chaque ligne de $N(x, t ; \xi)$ est le vecteur propre de ${ }^{t} H(x, t ; \xi)$, fixé par la condition que sa dernière composante soit égale à 1 .

Finalement, soit

$$
N(x, t ; \xi) H(x, t ; \xi)=D_{1}(x, t ; \xi) N(x, t ; \xi)
$$

où

$$
D_{1}(x, t ; \xi)=\left(\begin{array}{cccccc}
\lambda_{1}^{+} & & & & & \\
& \ddots & & & & \\
& \ddots & \lambda_{s}^{+} & & & \\
& & \lambda_{1}^{-} & & \\
& & & \ddots & \\
& & & \lambda_{s}^{-} & \\
& & & & \ddots
\end{array}\right) .
$$

Désignons

$$
N U_{n}={ }^{t}\left(w_{1}^{(n)}, \cdots, w_{s}^{(n)}, w_{s+1}{ }^{(n)}, \cdots\right)
$$

et posons

$$
S\left(t ; N U_{n}\right)=m \sum_{i=1}^{s}\left\|e^{-\varepsilon / \sqrt{\Lambda^{t}}} w_{i}^{(n)}\right\|^{2}-\sum_{j=s+1}^{m}\left\|e^{-\varepsilon_{1} / \overline{\Delta^{t}}} w_{j}{ }^{(n)}\right\|^{?}
$$

où $e^{-\varepsilon \sqrt{\Lambda t}}$ est défini par

$$
\left(e^{-\varepsilon} \sqrt{\Lambda^{t}} f(x)\right)^{\wedge}(\xi)=e^{-\varepsilon V} \overline{|\xi|^{t}} \hat{f}(\xi) .
$$

et on choisit $\varepsilon>0$ petit, par exemple, $\varepsilon<\frac{\delta^{\prime}}{3}$. 
Finalement posons

(6. 5) $\quad U_{n \rho}={ }^{t}\left((\Lambda+1)^{m-1}\left[\alpha_{n}^{(\rho)}(D) u\right],(\Lambda+1)^{m-2} \partial_{1}\left[\alpha_{n}^{(\rho)} u\right], \cdots, \cdots\right)$.

Un a la

Proposition 6. 1.

$$
\begin{aligned}
& \frac{d}{d t} S\left(t ; N U_{n}\right) \geqslant \delta^{\prime \prime} \sqrt{n}\left\|e^{-\varepsilon \sqrt{\Lambda^{t}}} N U_{n}\right\|^{2}-c_{1} \sqrt{n} \sum_{1 \leqslant|\rho| \leqslant k}\left\|e^{-\varepsilon \sqrt{\Lambda^{t}}} U_{n, \rho}\right\|^{2} \\
& -\frac{c_{2}}{\sqrt{n}}\left\|e^{-\varepsilon \sqrt{\Lambda^{t}}} f\right\|^{2}-\frac{c_{3}}{n^{2(k-l)}}\|u\| \|^{2},
\end{aligned}
$$

où $k(>l)$ est un nombre entier qu'on peut choisir aussi grand qu'on le veut, et $\delta^{\prime \prime}, c_{1}, c_{2}, c_{3}$ sont des constantes positives indépendantes de $u(x, t)$ et de $n$.

Comme la démonstration est assez longue, nous la donnerons dans un article ultérieur.

Revenons à (5.7). Désignons

(6. 6) $U_{n, \rho \sigma}={ }^{t}\left((\Lambda+1)^{m-1}\left[\alpha_{n}^{(\rho)} \beta^{(\sigma)} u\right],(\Lambda+1)^{m-2} \partial_{1}\left[\alpha_{n}^{(\rho)} \beta^{(\sigma)} u\right], \cdots\right)$.

Un calcul délicat montre

$$
\begin{aligned}
& \left\|e^{-\varepsilon \sqrt{\Lambda^{t}}} Q_{\rho \sigma}\left[\alpha_{n}^{(\rho)} \beta^{(\sigma)} u\right]\right\|^{2} \\
& \quad \leqslant c n \sum_{\mid \rho+\rho^{\prime} i \leqslant k} n^{\mid \rho \prime} \mid\left\|e^{-\varepsilon \sqrt{\Lambda^{t}}} N U_{n, \rho+\rho^{\prime} \sigma}\right\|^{2}+\frac{c^{\prime}}{n^{2(k-l-2)}}\|u\| \|^{2} .
\end{aligned}
$$

Compte tenu de la Proposition 6.1, on a

Proposition 6.2.

$$
\begin{aligned}
& \frac{d}{d t} S\left(t ; N U_{n}\right) \geqslant \delta^{\prime \prime} \sqrt{n}\left\|e^{-\varepsilon V \overline{\Lambda^{t}}} N U_{n}\right\|^{2} \\
& \quad-c \sqrt{n} \sum_{(\rho, \sigma)} n^{|\rho|-|\sigma|}\left\|e^{-\varepsilon V \bar{\Lambda} t} N U_{n, \rho \sigma}\right\|^{2}-\frac{c^{\prime}}{n^{2(k-l-2)}}\|u\|^{2},
\end{aligned}
$$

où la sommation est étendue $\dot{a}$ tout $(\rho, \sigma)$ satisfaisant $\dot{a}|\sigma| \leqslant m$, $|\rho| \leqslant k$, et $|\rho+\sigma| \geqslant 1$.

On considère, au lieu de (5.7), l'équation en $\alpha_{n}^{(\rho)} \beta^{(\sigma)} u$ :

$$
P\left[\alpha_{n}^{(\rho)}(D) \beta^{(\sigma)} u\right]=\sum_{\substack{1 \leqslant\left|\rho^{\prime}, \sigma^{\prime}\right| \leqslant k \\\left|\sigma^{\prime}\right| \leqslant m}} Q_{\rho^{\prime} \sigma^{\prime}}\left[\alpha_{n}^{\left(\rho+\rho^{\prime}\right)} \beta^{\left(\sigma+\sigma^{\prime}\right)} u\right]+R_{n, \rho \sigma}[u] .
$$

En posant

$$
\Theta_{n}(\rho, \sigma) u(x, t)=\sqrt{n}^{|\rho|-\mid \sigma i} e^{-\varepsilon / \overline{\Lambda t}} N U_{n, o \sigma}
$$


on a

Proposition 6. 3.

$$
\begin{aligned}
& \frac{d}{d t} S\left(t ; \Theta_{n}(\rho, \sigma) u\right) \geqslant \delta^{\prime \prime} \sqrt{n}\left\|_{n}(\rho, \sigma) u\right\|^{2} \\
& \quad-c \sqrt{n} \sum_{\left(\rho^{\prime}, \sigma^{\prime}\right)}\left\|\Theta_{n}\left(\rho^{\prime}, \sigma^{\prime}\right) u\right\|^{2}-\frac{c n^{k}}{n^{2(k-l-2)}}\|u\| \|^{2},
\end{aligned}
$$

ò la sommation est étendue à tout $\left(\rho^{\prime}, \sigma^{\prime}\right)$ satisfaisant $\dot{a} \rho^{\prime} \geqslant \rho, \sigma^{\prime} \geqslant \sigma$, et $|\rho+\sigma|+1 \leqslant\left|\rho^{\prime}+\sigma^{\prime}\right| \leqslant k$.

Posons

$$
S_{n}(t ; u(x, t))=\sum_{0 \leqslant|\rho+\sigma| \leqslant k} M^{|\rho+\sigma|} S\left(t ; \Theta_{n}(\rho, \sigma) u\right) .
$$

Si l'on prend $M$ assez grand, on a

\section{Proposition 6. 4.}

$$
\frac{d}{d t} S_{n}(t ; u(x, t)) \geqslant \delta^{\prime \prime \prime} \sqrt{n} S_{n}(t ; u(x, t))-\frac{c}{n^{k-2 l-4}}\|\| u\|\|^{2}
$$

7. Supposons que le problèmende Cauchy soit bien posé. Définissons $\hat{\psi}(\xi) \neq 0$, continue, dont le support est contenu dans un petit voisinage de $\xi_{0}$. On suppose de plus que, sur le support de $\hat{\psi}(\xi)$, $\alpha(\xi) \equiv 1$. Notons $\psi(x)=\mathscr{F}^{-1}[\hat{\psi}(\xi)]$.

Définissons $u_{n}(x, t)$ comme la solution de

$$
P\left[u_{n}(x, t)\right]=0
$$

avec

$$
\begin{aligned}
& u_{n}(x, 0)=\frac{\partial}{\partial t} u_{n}(x, 0)=\cdots=\frac{\partial^{m-2}}{\partial t^{m-2}} u_{n}(x, 0)=0 \\
& \frac{\partial^{m-1}}{\partial t^{m-1}} u_{n}(x, 0)=e^{i n x \xi_{0}} \psi(x) .
\end{aligned}
$$

D'après l'hypothèse, il existe des constantes positives $C$ et $p$ telles que

$$
\left\|\left|u_{n}(x, t)\right| i|\leqslant C|\right\| u_{n}(x, 0) \mid \|_{p} \leqslant C^{\prime} n^{p} \quad(n=1,2, \cdots) .
$$

Si l'on prend dans (6.9), $u(x, t)=u_{n}(x, t)$ et qu'on prend $k$ de telle manière que 


$$
k=2 l+5+2 p
$$

on aura

$$
S_{n}{ }^{\prime}\left(t ; u_{n}(x, t)\right) \geqslant \delta^{\prime \prime \prime} \sqrt{n} S_{n}\left(t ; u_{n}(x, t)\right)-0\left(\frac{1}{n}\right) .
$$

D'ailleurs, on a

$$
S_{n}\left(0 ; u_{n}(x, 0)\right) \geqslant c(>0) \text { pour } n>N \text { (assez grand). }
$$

Ces deux inégalités entraînent que

$$
S_{n}\left(t ; u_{n}(x, t)\right) \geqslant \frac{c}{2} \exp \left(\delta^{\prime \prime \prime} \sqrt{n} t\right),
$$

pour $n$ assez grand. Or, cette inégalité est contradictoire avec (7.1). Notre démonstration du Théorème 4 est donc achevée.

\section{Bibliographie}

[1] Hörmander, L., Pseudo-differential operators, Comm. Pure Appl. Math. 18 (1965), 501-158.

[2] Kano, T., On the Cauchy problem for equations with multiple characteristics, à paraitre au J. Math. Soc. Japan.

[3] Kohn, J. J. and L. Nirenberg, An algebra of pseudo-differential operators, Comm. Pure Appl. Math. 18 (1965), 269-305.

[4] Lax, A., On Cauchy's problem for partial differential equations with multiple characteristics, Comm. Pure Appl. Math. 9 (1956), 135-169.

[5] Leray, J. et Y. Ohya, Equations et systèmes non linéaires, hyperboliques non stricts, Math. Ann. 170 (1967), 167-205.

[6] Levi, E. E., Caratteristiche multiple e problema di Cauchy, Ann. di Mat. 16 (1909), 161-201.

[7] Matsumura, M., Existence locale de solutions pour quelques systèmes d'équations aux dérivées partielles, Jap. J. Math. 32 (1962), 13-49.

[8] Mizohata, S., Lectures on the Cauchy problem, Tata Institute of Fundamental Research, 1962.

[9] , Some remarks on the Cauchy Problem, J. Math. Kyoto Univ. 1 (1961-1962), 109-127.

[10] Ohya, Y., Le problème de Cauchy pour les équations hyperboliques à caractéristique multiple, J. Math. Soc. Japan, 16 (1964), 268-286.

[11] Yamaguti, M., Le problème de Cauchy et les opérateurs d'intégrale singulière, Mem. Coll. Sci. Kyoto Univ. 32 (1959), 121-151. 